\title{
Empirical Evaluation of OI-MAC: Direct Interconnection between Wireless Sensor Networks for Collaborative Monitoring
}

\author{
Krongboon Singhanat, Teng Jiang, Geoff V. Merrett, Nick R. Harris \\ Electronics and Computer Science, University of Southampton, UK SO17 1BJ \\ Email:\{ks14g13,tj2g11,gvm,nrh\}@ecs.soton.ac.uk
}

\begin{abstract}
Cooperation between co-located Wireless Sensor Networks (WSNs) has the potential to present new opportunities for novel applications and provide network performance improvements. The traditional interconnection approach for WSNs is based on a backbone network such as the Internet, but this may have intermittent or unavailable connectivity in remote locations. To address this, Opportunistic Direct Interconnection (ODI) has been proposed to allow distinct and independent WSNs to communicate directly with neighbouring networks, and OIMAC is a link-layer protocol which implements this functionality. However, OI-MAC has not been experimentally validated, instead with analysis performed through simulation. In this paper, we present a practical implementation of OI-MAC using two separate multi-hop networks with 6 sensor nodes in each. We validate its effective operation through experimentally obtained timing diagrams, sensor data output, and energy consumption. Results show successful cross-network packet communication, while networks remain independent by maintaining individual configurations and communication channels. Furthermore, we show that the process of discovering neighbouring networks has an insignificant impact on energy consumption.
\end{abstract}

\section{INTRODUCTION}

Wireless Sensor Networks (WSNs) provide significant benefits to many monitoring applications. Over time, a side-effect of their increasing popularity is an increase in the likelihood of multiple WSNs being spatially co-located (i.e. different networks having overlapping radio coverage). As a single WSN has constrained resources and a fixed coverage area, some applications have leveraged multiple networks to support a monitoring task. The trend towards pervasive computing and the Internet of Things motivates research on cooperation between co-located networks. Recent research has shown that the network lifetime of distinct co-located networks can be prolonged by cooperative packet forwarding [1]-[3]. Strategies to determine interactions between neighbouring networks have been proposed as both centralised [4] and distributed [5] algorithms. A software framework to combine data from separate networks has been introduced by enabling service composition between cooperative networks [6]. By exploiting cooperation between distinctive networks, benefits can be obtained either in terms of information-sharing or in terms of network-resource sharing [7]-[10].

To clearly illustrate this benefit, consider the application of water quality monitoring. Across a water catchment, many different monitoring networks will be used, each with different and independent stakeholders. For example, monitoring of surface water may be deployed by governments, while farmowners may deploy networks to manage irrigation for precision agricultural. All stakeholders can benefit from knowledge of parameters outside of the bounds of their own network. For example, a farmer's field is likely to be impacted by events occurring in neighbouring fields, such as irrigation or fertilisation. Knowledge of this can enhance a control strategy, e.g. reducing irrigation after detecting that a neighbouring field will shortly be generating useful run-off. Hence, in order to deliver an effective catchment-scale system, all individual and independent networks need to cooperate with each other [11].

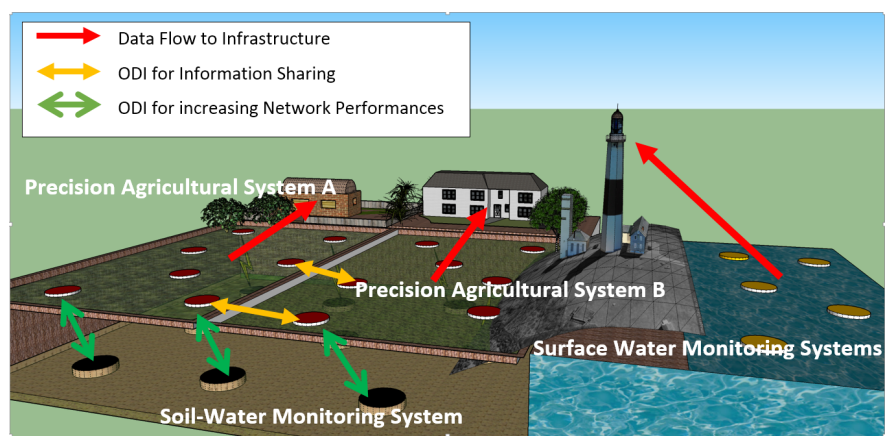

Fig. 1: Collaborative catchment-scale monitoring of precision agricultural and water quality control systems

In traditional schemes, interconnection between separate network entities is achieved using backbone networks (for example, LAN, WAN, MAN or the Internet). However, inaccessible and remote areas (which are often synonymous with the environments relevant to catchment-scale monitoring), hazardous environments, or spontaneous deployment can render backbone infrastructures infeasible, preventing cooperation. To solve this problem, Opportunistic Direct Interconnection (ODI) has been proposed [8] for establishing cooperation between co-located (neighbouring) WSNs. Figure 1 illustrates the potential for ODI to enable catchment-scale monitoring, by combining data contributed from multiple networks. Here, we illustrate an underground wireless sensor network utilising ODI to inject its packets into WSNs on the surface, improving link quality/energy consumption when compared to direct communication with a single central point located on the surface.

Two features are required to implement ODI. First is a method of achieving Direct Interconnection whereby different heterogeneous networks are able to communicate with each 
other. This is particularly challenging as the requirements of different applications typically renders a single standardised communication stack and hardware platform infeasible. The second requirement is to provide Opportunistic Interconnection requiring the automatic run-time discovery of link-layer connections when neighbouring networks are detected. Such run-time discovery is essential as identifying all neighbouring networks (both those which currently present and those which will appear in the future) are impossible at design-time.

To solve these challenges, it is required that co-located WSNs share a certain degree of similarity. Increased adoption of IEEE 802.14.5 has resulted in greater compatibility between transceivers. However, the heterogeneity of higherlayers presents greater difficulty. OI-MAC [8] was proposed to provide link-layer support for ODI, incorporating network discovery, handshaking and cross boundary data exchange. However, OI-MAC has not been experimentally validated, with analysis instead performed through simulation.

In this paper, we present the first practical validation of ODI, by implementing an OI-MAC network using the Texas Instruments eZ430-RF2500 sensor nodes. Evaluation results show that co-located networks can discover each other opportunistically, and perform successful cross-boundary communication. The energy required to support OI-MAC's discovery scheme is shown to be insignificant compared to normal operation, and the energy overheads related to cross-boundary communication are minimal.

The remainder of this paper is organised as follows: In the next section, the basic concepts of OI-MAC are presented. Subsequently, section III presents details on how OI-MAC was practically implemented. The setup of the experimental validation is discussed in section IV, followed by the presentation and discussion of experimental results in section $\mathrm{V}$.

\section{OVERVIEW OF OI-MAC}

Conventional MAC protocols cannot support ODI because their design principle is to avoid potential interference from neighbouring networks by maintaining a virtual wall around their network (this could be implemented using different channels or different PAN IDs). This means that co-located networks typically cannot communicate directly with each other, even if they adopt the same protocol stack. OI-MAC extends a state-of-the-art MAC protocol (RI-MAC [12]), proposing additional functions to enable ODI. However, it is believed that OI-MAC's fundamental concepts could also be used to extend other state-of-the-art protocols.

OI-MAC is the first MAC protocol designed to include functionality related to ODI (discovery and cross-boundary transmission) as part of the protocol logic. As a receiverinitiated multi-channel MAC protocol, OI-MAC reserves one channel as a Common Channel $(\mathrm{CCH})$ for discovery and handshaking, while the other channels are treated as Data Channels (DCH) for use by independent networks. Neighbouring networks adopting OI-MAC utilise different DCHs in order to avoid interference and maintain their network independence. The process of discovering neighbouring networks is performed by a combination of two modes: active and passive discovery (as shown in Figure 2).

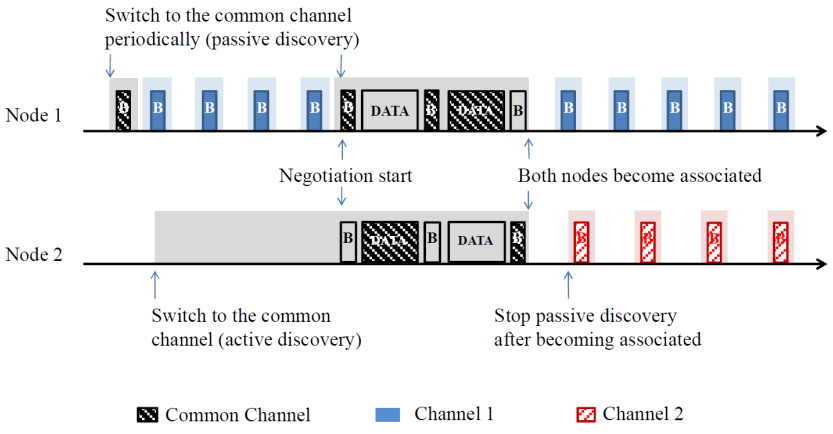

Fig. 2: The theoretical timing diagram of OI-MAC's discovery scheme (reproduced from [8])

Passive discovery requires each node to switch to the $\mathrm{CCH}$ periodically (as defined by the discovery period) and broadcast a discovery beacon that contains the network ID and DCH. Once the broadcast is completed, the node listens (for a period defined by the dwell time) and enters into a handshaking process if there are any replies.

During active discovery, each sensor node switches to the $\mathrm{CCH}$ and listens for the entire duration of a discovery period. If a discovery beacon is received, the node responds immediately and begins the handshaking process. During this process, both transmitters exchange details (for example the DCH frequency, network ID and wakeup period) and become associated with one another as Boundary Nodes (BNs). After handshaking, the $\mathrm{BN}$ is considered as an additional data sink (a central point of data collection). A node performs cross-boundary data injection (i.e. injecting packets into the neighbouring network) by switching its transceiver to the $\mathrm{DCH}$ of the neighbouring network and transmitting packets.

\section{IMPLEMENTATION OF OI-MAC}

To validate the concept of ODI for allowing cooperation between independent WSNs, we have practically implemented OI-MAC on real hardware. This allows us to experimentally observe and evaluate packet communication, protocol timing, and energy consumption.

Our deployment platform is the Texas Instruments eZ430RF2500 [13], consisting of a low-power MSP430F2274 microcontroller (supporting a range of energy-efficient sleep states), a $2.4 \mathrm{GHz}$ CC2500 radio transceiver, and an on-board/on-chip temperature sensor.

The OI-MAC sleep period is set to a duration of $256 \mathrm{~ms}$. Furthermore, the OI-MAC protocol suggests that a pseudorandom sleep period is intentionally generated to avoid local synchronisation of wake-up schedules, which will result in collisions. In the low-power polling state, the radio receiver remains active for $20 \mathrm{~ms}$, waiting for an incoming packet. This results in a duty cycle of 7.8 percent. For cross boundary communication, the active discovery interval is set at 20 seconds, while passive discovery occurs once every 60 sleep periods (approximately once every 15 seconds, thus ensuring overlap between active and passive discovery).

Packet routing is implemented using a tree-based routing algorithm, as commonly used in data-centric WSNs. The root 
of the tree is the sink node(s). At route setup, routes are built using gradients to the sink node(s). Longer routes are characterised by higher gradient values, and hence routing via nodes with lower gradient values selects the shortest path to the sink. As ODI introduces multiple data sinks (both the network's own sink node and the BNs), the routing protocol establishes and records multiple routes by introducing an additional gradient at each node. The gradient of each node can change dynamically, dependent on the link quality.

The application running on the network periodically samples the temperature sensor every 2.5 seconds. After processing the raw signal and forming it into a packet, it is added to the transmit queue. Generated packets are transmitted to either the local network or the neighbouring network. The decision of which to transmit to is randomly decided such that a required average injection ratio $(\alpha)$ is achieved. The injection ratio is defined by:

$$
\alpha=\frac{\text { Number of injected packets }}{\text { Total number of generated packets }}
$$

Varying the injection ratio allows us to compare the network performance and overheads of cross-boundary communication compared to regular network traffic.

\section{EXPERIMENTAL SETUP}

To evaluate the operation of OI-MAC, a simple network architecture consisting of two distinct networks is considered (see Figure 3). Each network uses a different DCH to communicate, and each has its own sink node that is connected to a PC via UART.

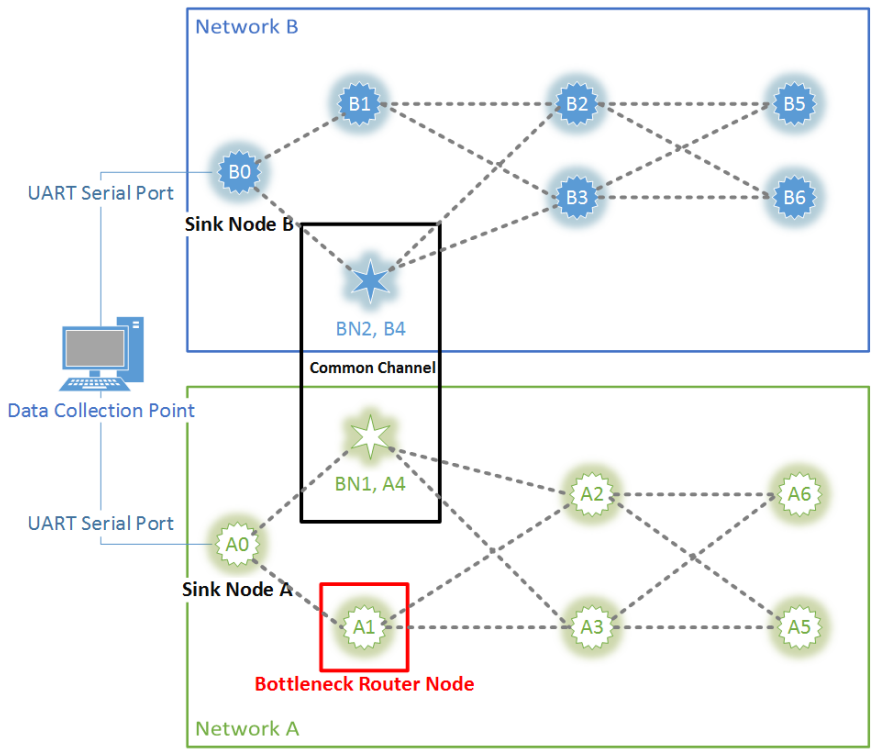

Fig. 3: The network architecture used in the experimental evaluation.

A routing node, $\mathrm{A} 1$, is intentionally placed at a distance nearest to its sink in order to ensure that it becomes the router node in this bottleneck area. Nodes capable of cross-boundary communication are a single hop from their sink to enable a comparison between the $\mathrm{BNs}$ and the router node in the bottleneck area.

\section{EXPERIMENTAL RESULTS}

This section presents the results from our experimental validation of OI-MAC. First, correct functional operation of OI-MAC is evaluated. The injection ratio $\alpha$ is set to 0.5 (i.e. half of the packets generated by Network A are destined for Sink Node A, while the other half are destined for Sink Node B). The packets received by both Sink Nodes from Node A6 are shown in Figure 6. This clearly illustrates that the packets are correctly and timely received regardless of whether they were communicated internally within their own network, or injected into the neighbouring network.

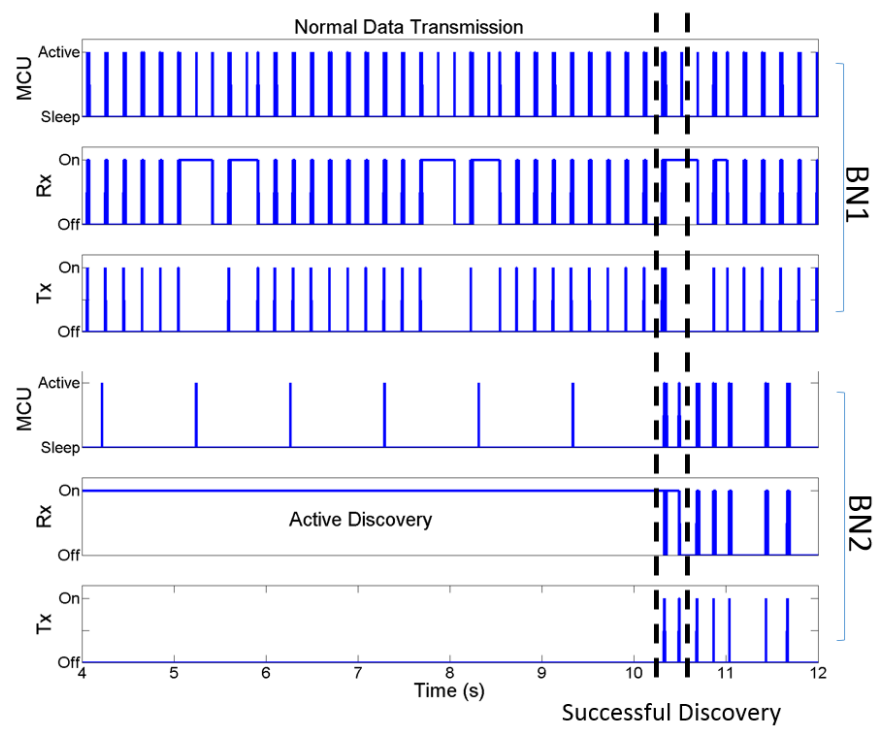

Fig. 4: Experimentally obtained timing diagram illustrating successful neighbour discovery.

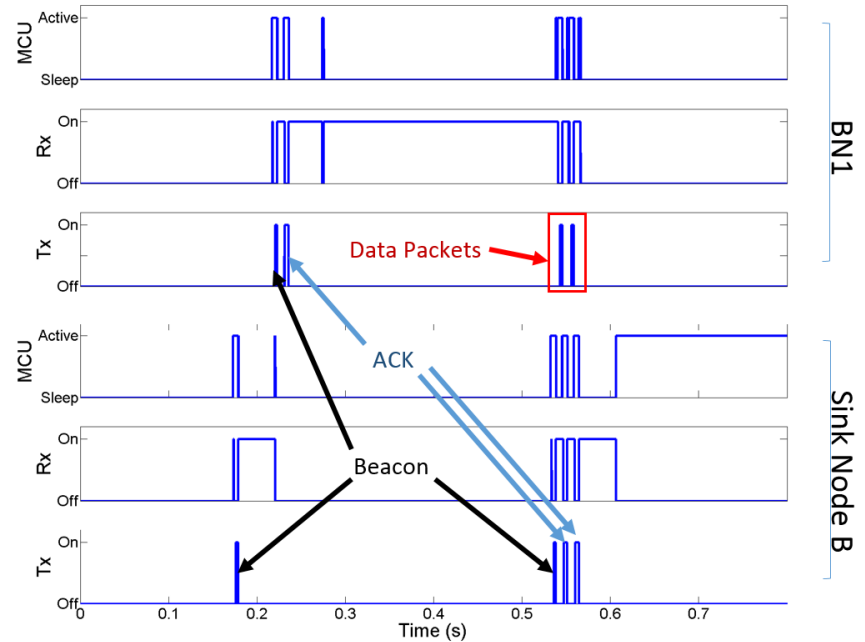

Fig. 5: Experimentally obtained timing diagram showing the injection of data packets in the neighbouring network. (Data packet reception can be inferred from the subsequent transmission of an acknowledgement.)

Figures 4 and 7 illustrate the state of the MCU and radio transceiver during the discovery process. Rx channel indicates 


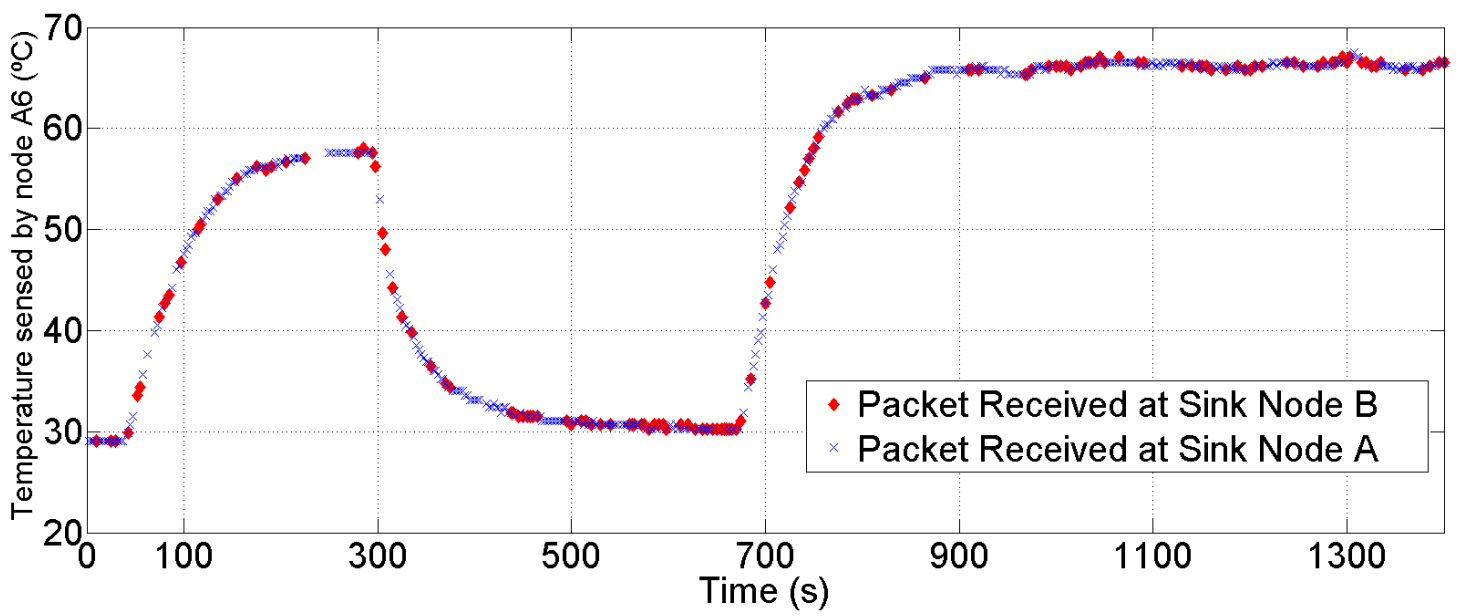

Fig. 6: Temperature samples originating from node A6, as captured by Sink Node A and B

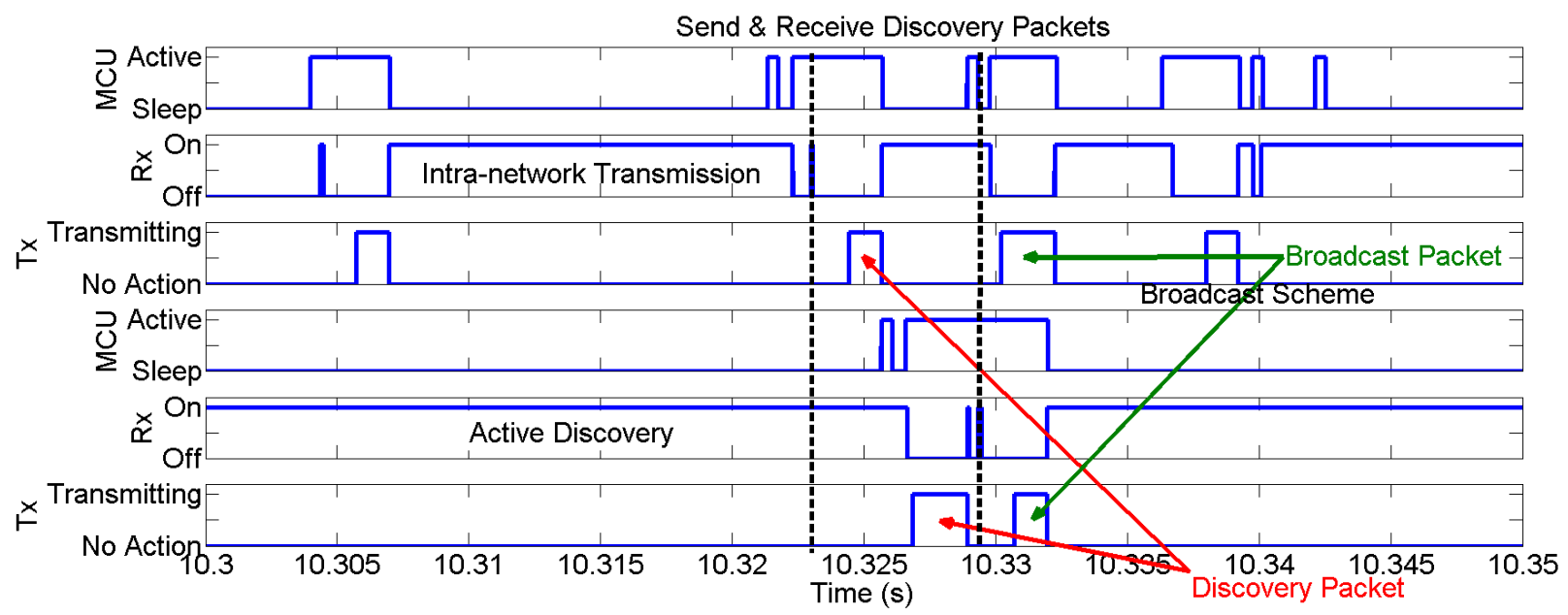

Fig. 7: Experimentally obtained timing diagram showing the transmission and reception of discovery packets and broadcasts. At $T=10.3225$, Node BN1 begins passive discovery. Both BNs exchange configuration data by sending and receiving discovery packets and broadcasting the successful discovery.

when the receiver is in receive mode. Figure 4 displays the case where co-located networks detect each other, and both communicate via the common channel. In this timing diagram, BN1 performs its normal operation and periodically switches its transceiver to the common channel for passive discovery. At around 10.2 seconds, the discovery attempt is successful because the discovery packet is received by BN2. After receiving the discovery packet, BN2 replies with another discovery packet containing information on its own network configuration. Subsequently, both networks initiate a new broadcast to inform surrounding nodes in the network. This process can be clearly seen in Figure 7.

Figure 5 shows the injection of data packets into the neighbouring network. After ODI has been established, BN1 performs an additional role in transmitting data packets across the network boundary. Each cycle, BN1 switches its transceiver to the DCH of the neighbouring network. In the case shown in Figure 5, BN1 receives a data packet from a node in Network A. Subsequently, BN1 transmits two data packets across the boundary to Sink Node B, which responds to each packet with an ACK for each.

\section{A. Energy Consumption}

In the experimentally obtained timing diagrams shown in Figures 7 and 4, the MCU spends most of its time in the sleep state, only waking up from a radio interrupt in order to process packets or sensor data for a short time period (in the order of micro seconds). This suggests that the major consumers of energy are radio communication tasks. Figure 8 shows the energy consumption of different nodes over a 10 minute period. Data were obtained using an Agilent N6705B DC Power Analyzer, allowing accurate seamless measurement of both sleep and active currents, and analysis of the results.

TABLE I: Average power and energy consumption of RN and BNs in cross boundary transmission scheme

\begin{tabular}{|c||c||c|}
\hline Node & avg. Power (mW) & Energy Consumption (J) \\
\hline RN ("Bottleneck") & 23.87 & 14.32 \\
\hline BN (Passive Discovery) & 22.64 & 14.64 \\
\hline BN $(\alpha=0.5)$ & 25.55 & 15.78 \\
\hline BN $(\alpha=1)$ & 27.67 & 17.39 \\
\hline
\end{tabular}




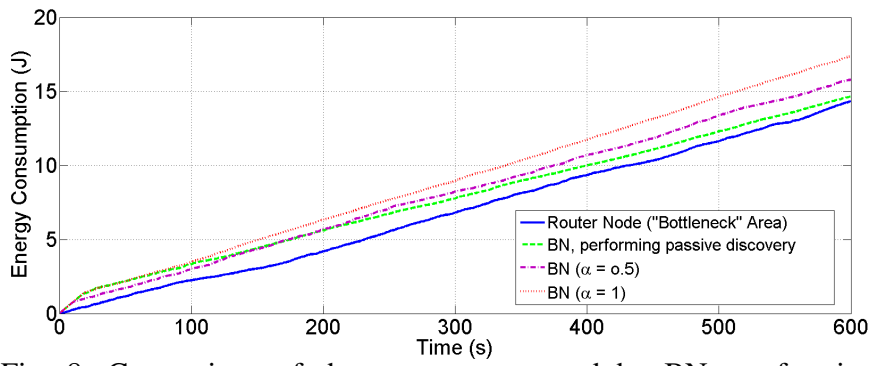

Fig. 8: Comparison of the energy consumed by BNs performing different amounts of packet injection, compared against a normal router node

In Figure 8, the energy consumed by active discovery can be seen at the beginning of the traces. All BNs incur this initial energy cost, caused by the continuous reception required for active discovery. However, as active discovery is only performed once in a network's life, this cost is comparatively insignificant over a prolonged period of operation. The operation of the node after active discovery has a more significant effect on the node's lifetime. The average power consumptions (see Table I), depicted by the gradient of the energy consumption, highlights that passive discovery does not shorten the overall network lifetime as BNs with no crossboundary connections consume less energy than the routing node (RN) in the "bottleneck" area. This is because BNs are typically on the periphery of the network (leaf-nodes in the routing tree), and so do not otherwise have considerable packet throughput. With $\alpha=0.5$, the average power consumption of the RN and $\mathrm{BN}$ are approximately equal. As expected, when $\alpha$ is increased to 1 (where all packets are injected into the neighbouring network), the average power consumption of the $\mathrm{BN}$ becomes marginally higher than the bottleneck router node (up to 11 percent).

\section{CONCLUSIONS}

Cooperation between WSNs has the potential to realise new applications and improved network performance. It has recently been proposed that such cooperation can be achieved by enabling networks to identify and connect directly with each other at run-time. A link-layer protocol, OI-MAC, has been proposed as a solution to enable this, but it has only been evaluated through simulation. In this paper, we have reported on a practical implementation of OI-MAC on two small networks. The experimentally obtained results illustrate that OI-MAC can be practically implemented and operate as designed. They also illustrate that the energy consumption overheads are acceptable, and are in agreement with those suggested in the existing simulation results. Further validation and comparison of our experimental results against simulations will be included in our future work.

\section{REFERENCES}

[1] N. Inoue, K. Kinoshita, T. Watanabe, K. Murakami, Y. Tanigawa, and $\mathrm{H}$. Tode, "A cooperative routing method with shared nodes for overlapping wireless sensor networks," 2014 International Wireless Communications and Mobile Computing Conference (IWCMC), pp. 1106-1111, Aug. 2014. [Online]. Available: http://ieeexplore.ieee.org/lpdocs/epic03/wrapper.htm?arnumber=6906509

[2] K. Bicakci and B. Tavli, "Prolonging network lifetime with multidomain cooperation strategies in wireless sensor networks," Ad Hoc Networks, vol. 8, no. 6, pp. 582-596, Aug. 2010. [Online]. Available: http://linkinghub.elsevier.com/retrieve/pii/S1570870509001164

[3] T. Jiang, G. V. Merrett, and N. R. Harris, "Enabling Opportunistic Energy Trading between Overlapping Energy Harvesting Wireless Sensor Networks," pp. 0-5, 2014.

[4] M. Rovcanin, E. D. Poorter, I. Moerman, and P. Demeester, "A reinforcement learning based solution for cognitive network cooperation between co-located, heterogeneous wireless sensor networks," Ad Hoc Networks, vol. 17, pp. 98-113, Jun. 2014. [Online]. Available: http://linkinghub.elsevier.com/retrieve/pii/S1570870514000201

[5] V. Jeličić, D. Tolić, and V. Bilas, "Consensus-based decentralized resource sharing between co-located Wireless Sensor Networks," in IEEE ISSNIP 2014 - 2014 IEEE 9th International Conference on Intelligent Sensors, Sensor Networks and Information Processing, Conference Proceedings, no. April, 2014, pp. 21-24.

[6] T. De Pauw, B. Volckaert, A. Hristoskova, V. Ongenae, and F. De Turck, "Symbiotic Service Composition in Distributed Sensor Networks," International Journal of Distributed Sensor Networks, vol. 2013, pp. 1-22, 2013. [Online]. Available: http://www.hindawi.com/journals/ijdsn/2013/684563/

[7] E. Poorter, B. Latré, I. Moerman, and P. Demeester, "Symbiotic Networks: Towards a New Level of Cooperation Between Wireless Networks," Wireless Personal Communications, vol. 45, no. 4, pp. 479495, Mar. 2008.

[8] T. Jiang, G. V. Merrett, and N. R. Harris, "Opportunistic Direct Interconnection between Co-Located Wireless Sensor Networks," 2013 22nd International Conference on Computer Communication and Networks (ICCCN), pp. 1-5, Jul. 2013.

[9] B. Karaoglu, I. Demirkol, and W. Heinzelman, "Exploring the Benefits of Symbiotic Routing," 2011 Proceedings of 20th International Conference on Computer Communications and Networks (ICCCN), pp. 1-6, Jul. 2011.

[10] T. Jiang, G. V. Merrett, and N. R. Harris, "Opportunistic energy trading between co-located energy-harvesting wireless sensor networks," Proceedings of the 1st International Workshop on Energy Neutral Sensing Systems - ENSSys '13, pp. 1-6, 2013.

[11] H. Zia, N. R. Harris, and G. V. Merrett, "The impact of agricultural activities on water quality: A case for collaborative catchment-scale management using integrated wireless sensor networks," Computers and Electronics in Agriculture 96, 2013.

[12] Y. Sun, O. Gurewitz, and D. Johnson, "RI-MAC: a receiver-initiated asynchronous duty cycle MAC protocol for dynamic traffic loads in wireless sensor networks," Proceedings of the 6th ACM conference on Embedded network sensor systems, pp. 1-14, 2008.

[13] “eZ430-RF2500 Development Tool User's Guide," 2009. [Online]. Available: http://www.ti.com/lit/ug/slau176d/slau176d.pdf 